\title{
P064
}

\section{Numerical and Experimental Study of Hydraulic} Fracture Active Source Monitoring

\author{
A. Nabipour (Curtin University), B. Evans (Curtin University), T. Mueller* \\ (CSIRO) \& M. Sarmadivaleh (Curtin University)
}

\section{SUMMARY}

Hydraulic fracturing is one of the most common operations performed on oil and gas wells. As the hydraulic fracture propagation is so complex, monitoring techniques are used to determine the real-time geometry of the induced fracture. In this work focus is made on numerical and experimental study of active monitoring of hydraulic fracture. Discrete element method is used for numerical simulation of seismic wave transmission in a block of rock being hydraulically fractured. In this method the rock is modeled by an assembly of round particles. On the other hand the results of an ultrasonic laboratory experiment in which a block of cement is fractured, are reported. Numerical and experimental deliver similar results which are in agreement with those published in literature. The results show interesting information which can be applied for active monitoring of field hydraulic fractures. 


\section{Vienna ''11}

\section{Introduction}

Hydraulic fracturing is a common stimulation operation performed on oil and gas wells to maximize the contact area between the wellbore and the reservoir. Due to many factors affecting the hydraulic fracturing operation, it is generally difficult to determine its final outcome. Microseismic monitoring methods are usually used for monitoring the extent of an induced hydraulic fracture. However, there are some limitations associated with such passive microseismic methods. For instance, the sources are not repeatable, the velocity field is often not simple, and it is not generally possible to correctly determine the width of a hydraulic fracture using these methods.

On the other hand, there is another category of monitoring methods in which the sources are active and repeatable. Vertical seismic profiling (VSP) and cross-well experiments are active-source methods which have been successfully tested in the field (Meadows and Winterstein, 1994; Wills et al. 1992). In the laboratory, the observed velocity dispersion of transmitted $P$ waves has been related to the width of the fracture (Groenenboom and Fokkema, 1998). Ultrasonic experiments performed later by Groenenboom and van Dam (2000) showed that wave diffractions caused by the fracture tip can be used as an indicator of the fracture extent. Numerical approaches are also extensively used for modelling the interaction of elastic waves with fractures. Although the most common methods used for modelling wave propagation are continuum mechanics-based numerical approaches such as finite difference method (FDM) and finite element method (FEM), in this study the discrete element method (DEM) is employed. In two dimensional DEM, the medium is simulated by an assembly of round rigid particles which can be bonded together. The macro-mechanical properties of such an assembly depend on the micro-properties of individual particles. An advantage of DEM over the other methods is that fracturing of material can be efficiently simulated. This method has been already shown to be capable of modelling elastic wave propagation for different arranged assemblies of particles (Toomey and Bean, 2000; Nabipour et al. 2011). Furthermore, Toomey et al. (2002) modelled wave propagation across a fracture using a particle-based scheme which resulted in good agreements with analytical methods.

In this study the result of numerical simulation of active seismic monitoring of a hydraulic fracture is presented. Particle Flow Code (PFC), a DEM-based numerical code is used for simulations (Itasca Consulting Group Inc, 2008). Afterwards, the observations made in a laboratory experiment of ultrasonic monitoring of hydraulic fracture propagation using a True Triaxial Stress Cell (TTSC) are presented. Numerical results are compared against those obtained from experiments.

\section{Numerical Modelling}

In this part the procedure for modelling hydraulic fracture monitoring in a $2 \mathrm{D}$ cubic packed assembly is briefly explained. More details can be found in Nabipour et al. (2011). A $100 \mathrm{~m} \times 100 \mathrm{~m}$ block of rock consisting of square packed particles of 1 meter diameter bonded together is generated and anisotropic horizontal in-situ stresses of $20 \mathrm{MPa}$ and $50 \mathrm{MPa}$ are applied to the sample. Employing the hydro-mechanical coupling capability of the code, an initial pore fluid pressure of $15 \mathrm{MPa}$ is set for the whole assembly. Then the pressure at the centre of the assembly is increased to $100 \mathrm{MPa}$ and the model is cycled with sufficient numerical steps such that fluid pressure propagation can be modelled. The increase in fluid pressure causes initiation and propagation of a symmetrical fracture by breaking the bonds between the particles. As expected, the fracture propagates parallel to the maximum horizontal stress direction. The distance between particle pairs on opposite walls of the fracture is continuously monitored and is used to construct the width profile of the fracture over the time. Over the injection period, seismic transmission is simulated between a source and a receiver as shown in Figure 1. Figure 2 shows the fracture width profile in different instances of simulation. Negative values indicate the overlap between particles due to compressional stress state. It is revealed that over the injection time the fracture width increases by forming a tapering shape. As can be seen the source-receiver line is intervened by the fracture at around 50 seconds after injection. 
Figure 3 shows the particle velocity $\mathrm{x}$-component at the receiver (corresponding to a P-wave) versus time which is recorded exactly before injection and in instants during the injection period. It is clear-

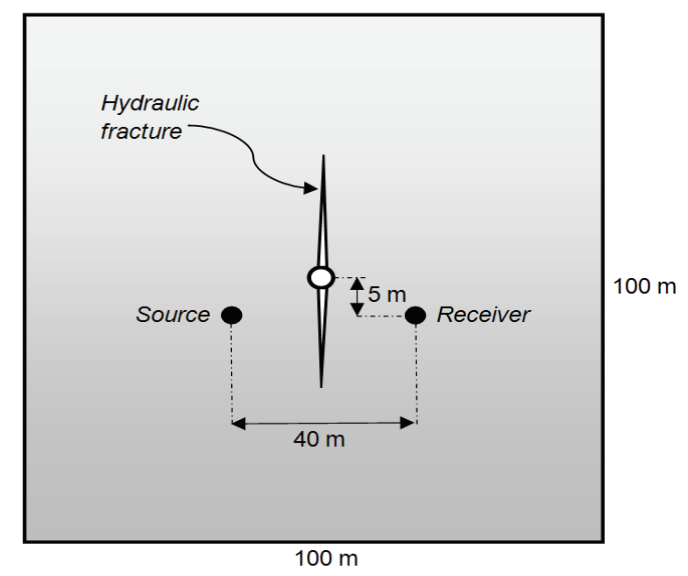

Figure1 Position of induced hydraulic fracture and seismic source/receiver in the numerical model.

Fracture width profile in different instances after starting injection

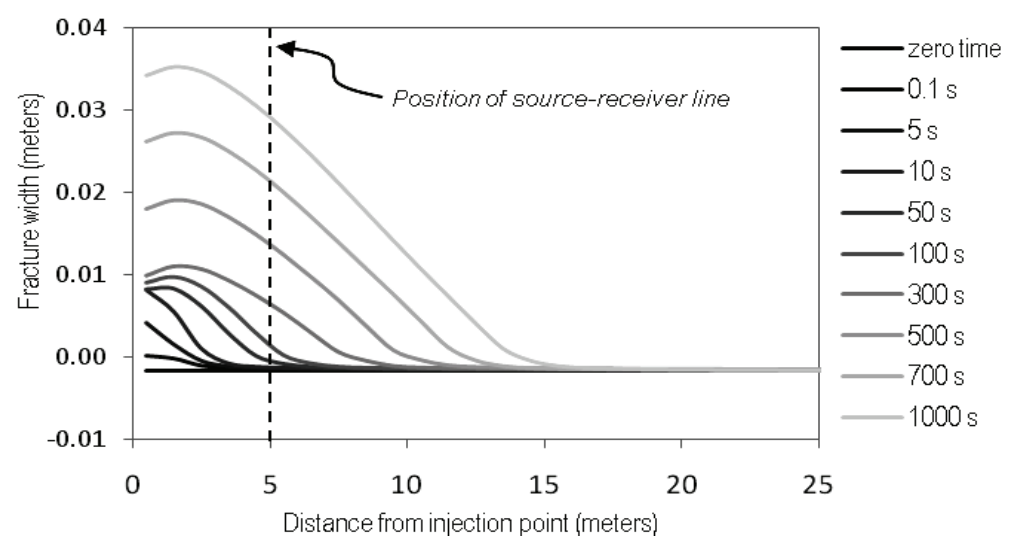

Figure2 Hydraulic fracture width versus distance from the centre of the model in different simulation instances.

-that after the start of injection and fracture initiation, a time delay occurs in the recorded waveforms. Recorded waveforms also indicate that the fracture intervention after 50 seconds of injection influences the time delay even more pronouncedly. The increasing delay and decreasing amplitude versus injection time correspond to a longer and wider fracture as shown by Figure 2. This shows that a smaller portion of wave energy reaches the receiver when the fracture interferes within the sourcereceiver path. These results are in good accordance with the experimental results obtained by Groenenboom and Fokkema (1998). Although the simulated hydraulic fracture is straight, this shows the capability of DEM in modelling seismic monitoring of such a fracture. Further useful information can be obtained by changing the properties of the model such as the particle packing scheme, fluid properties and micro-mechanical properties of the particles.

\section{Laboratory Experiment}

In order to model the process of active seismic monitoring of a field hydraulic fracture at the laboratory scale, an ultrasonic transmission experiment is performed using TTSC. By means of this facility three independent mechanical stresses can be applied on rock cubic samples. Furthermore, using computer-controlled injection pumps, arbitrary flow rates can be injected into the sample which causes the sample to fracture under high pressure. A $20 \mathrm{~cm}$ cube made of a mixture of cement, sand and water, set for one month in a water pool, is used as the test sample. A $7 \mathrm{~mm}$ diameter hole is drilled through the centre of the sample and one of its ends is plugged. The injection tube is inserted 


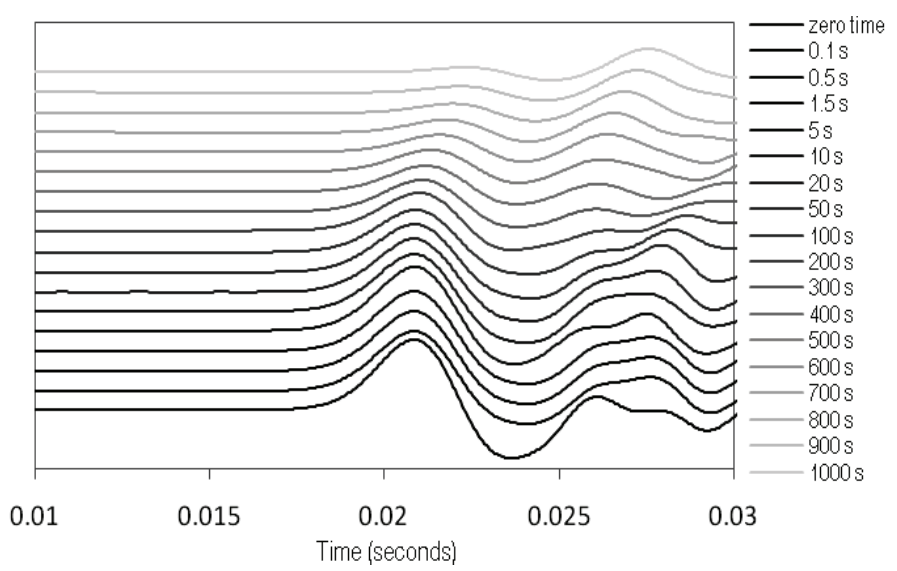

Figure 3 Waveform recorded before injection and in different instances over the injection period.

and glued to the other end of the drilled wellbore such that an open-hole section of $5 \mathrm{~cm}$ was left in the middle of the wellbore which makes initiation of the hydraulic fracture easier. The sample is set inside TTSC and a uniaxial horizontal stress of $17 \mathrm{MPa}$ is applied to two opposite surfaces of the sample to force propagation of the fracture in the desired direction. Two ultrasonic P-wave transducers are pushed against those opposite horizontal surfaces of the sample not experiencing any direct stresses. The hydraulic fracture is then expected to intervene in the the transducers' line in this configuration.

P-wave ultrasonic transmissions are performed before and during the injection of fracturing fluid inside the sample. The laboratory fracturing fluid has a viscosity of $100,000 \mathrm{cP}$ for scaling purposes. Fluid injection is started with a rate of $60 \mathrm{cc} / \mathrm{hr}$ into the sample. Ultrasonic transmissions with a frequency of $400 \mathrm{KHz}$ and a sampling rate of 54 scans per minute are performed during the injection. In addition, fracturing fluid pressure is recorded versus experiment time. Figure 4 shows the injection pressure versus time. It can be observed that after about 17 minutes of injection, the fracture initiates and starts to propagate. The peak stress reaches $4100 \mathrm{psi}$ and then drops suddenly. The injection is stopped one minute after the fracture initiation.

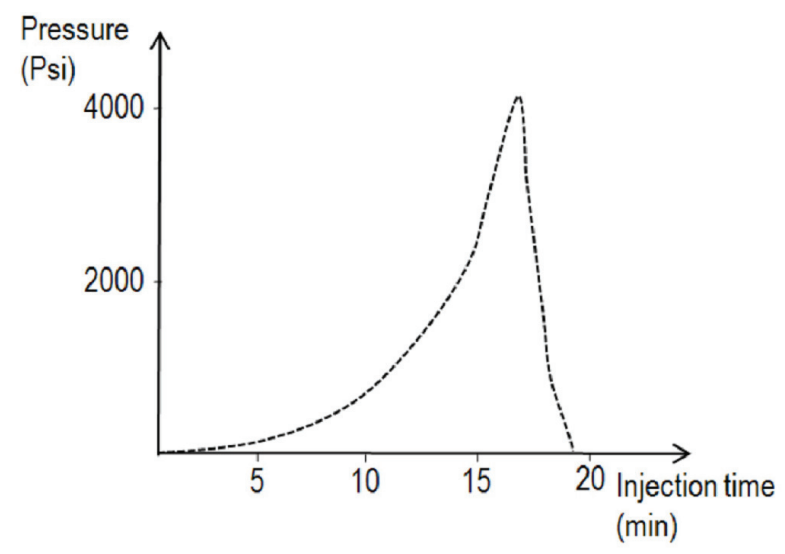

Figure 4 Fracturing fluid injection pressure versus experiment time.

Figure 5 shows transmission data of $\mathrm{P}$-wave recorded over the injection period (scan numbers 880 to 972). As expected, some variations are observed in the recorded data after the trace number 930, corresponding to the 17.2 minutes of injection time. This moment is close to the fracture initiation moment already obtained from Figure 4. The small difference indicates the time it took from the fracture to reach the transducers' line after its initiation. Looking at the graph, a reduction in the amplitude of the recorded waveforms is also observed. This is because less energy is transferred through the medium compared to the intact cement sample. When the hydraulic fracture opens and its walls become separated by the fluid force, the elastic wave has to pass two fluid-solid interfaces. 


\section{Vienna |'11}

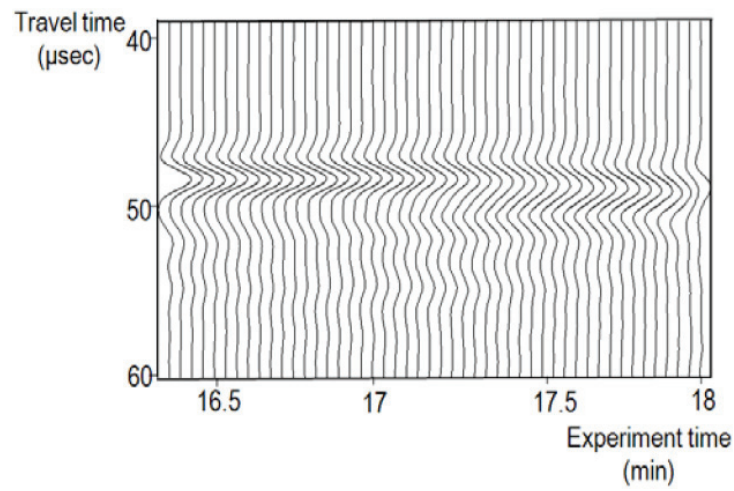

Figure 5 Recorded P-wave transmission data over the injection period.

This may reduce the portion of elastic energy transferred through the medium. Furthermore, the hydraulic fracture causes a delay in travel time of the ultrasonic waves. This can be explained by the smaller wave velocity in the fracturing fluid compared to the cement sample. It is expected that a wider hydraulic fracture causes stronger travel time delay. These results are very similar to the experimental data reported by Groenenboom and Fokkema (1998). As stated in their paper, such travel-time delay measurements can be used as an efficient indicator of the fracture width. Furthermore, DEM results are in good agreement with the experimental data indicating the capability of the numerical method for simulation of such phenomena.

\section{Conclusions}

Seismic monitoring of hydraulic fracturing is modelled using DEM. The method is capable of simulating elastic wave interaction with a hydraulically induced fracture. Numerical results show a time delay as well as an amplitude reduction when the fracture intervenes with the source-receiver path. These measurements can be used for determining the extent and width of the hydraulic fracture. Ultrasonic experiments performed showed similar results. The results are also comparable to those obtained in previous studies.

\section{References}

Groenenboom, J. and Fokkema, J.T. [1998] Monitoring the width of hydraulic fractures with acoustic waves. Geophysics, 63, 139-148

Groenenboom, J. and Van Dam, D.B. [2000] Monitoring hydraulic fracture growth: Laboratory experiments. Geophysics, 65(2), 603-611

Itasca Consulting Group Inc. [2008] Particle Flow Code in 2 Dimensions. Version 4.0 User's Manual. Minneapolis, Minnesota, USA.

Meadows, M.A. and Winterstein, D. F. [1994] Seismic detection of a hydraulic fracture from shearwave VSP data at Lost Hills Field California. Geophysics, 59 (1), 11-26

Nabipour, A., Evans, B., Müller, T. and Sarmadivaleh, M. [2011] Evaluation of PFC 2D for modelling seismic monitoring of hydraulic fracture. 2nd International FLAC/DEM Symposium.

Toomey, A. and Bean, C.J. [2000] Numerical simulation of seismic waves using a discrete particles scheme. Geophysical Journal International, 141, 595-604

Toomey, A., Bean, C.J. and Scotti, O. [2002] Fracture properties from seismic data- a numerical investigation. Geophysical Research Letters, 29(4), L013867

Wills, P.B., Demartini, D.C., Vinegar, H.J., Shlyapobersky, J., Deeg, W.F., Woerpel, J.C., Fix, J.E., Sorrells, G.G. and Adair, R.G. [1992] Active and passive imaging of hydraulic fractures. The Leading Edge, 11(7), 15-22 